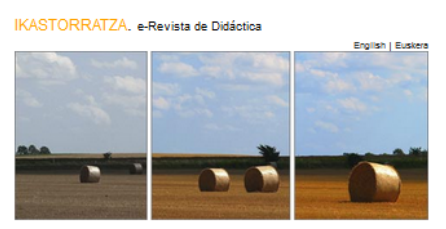

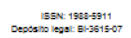

Inicio, Sobre nosotros, Publicacionesl, Participe

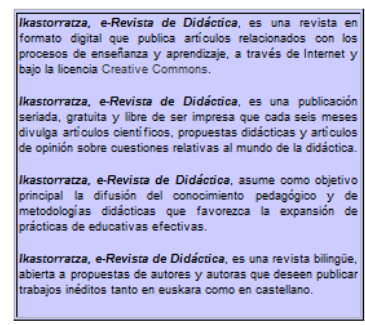

\section{IKASTORRATZA. Didaktikarako e-aldizkaria}

IKASTORRATZA. e-journal on Didactics

ISSN: 1988-5911 (Online) Journal homepage: http://www.ehu.eus/ikastorratza/

\title{
El interés del profesorado en formación en impartir nuevos contenidos históricos en Educación Primaria. Un caso de estudio en Madrid
}

\author{
IRASTORRATZA. e-Revista de \\ Didáctica
}

Esther Jiménez Pablo
ejimenezpablo@ucm.es

Gemma Muñoz García

gemunoz@ucm.es

To cite this article:

Jiménez, E. \& Muñoz, G. (2021). El interés del profesorado en formación en impartir nuevos contenidos históricos en Educación Primaria. Un caso de estudio en Madrid. IKASTORRATZA. e-Revista de Didáctica, 26, 73-95. DOI: 10.37261/26_alea/4

To link to this article:

https://doi.org/10.37261/26_alea/4

Published online: 31 March 2021 


\title{
El interés del profesorado en formación en impartir nuevos contenidos históricos en Educación Primaria. Un caso de estudio en Madrid
}

\section{The interest of trainee teachers in teaching new historical content in Primary Education. A case study in Madrid}

\author{
Esther Jiménez Pablo y Gemma Muñoz García ${ }^{2}$ \\ ${ }^{1}$ Universidad Complutense de Madrid \\ ejimenezpablo@ucm.es \\ ${ }^{2}$ Universidad Complutense de Madrid \\ gemunoz@ucm.es
}

\section{Resumen}

La presente investigación plantea un análisis de los contenidos históricos que le gustaría impartir al profesorado en formación del grado de Educación Primaria cuando ejerza como maestro/a en un colegio. Primero se estudia el impacto de las nuevas corrientes historiográficas en la enseñanza de la Historia, para luego analizar si estas tienen interés para el futuro profesorado. A través de una muestra de 118 participantes de la Universidad Complutense de Madrid, con formularios que ofrecen datos cuantitativos y cualitativos, se muestran datos significativos sobre el futuro de la enseñanza de la Historia. Los resultados revelan una profunda desconexión entre los contenidos curriculares presentes y la motivación de los estudiantes para enseñar determinados temas, siendo los contenidos de resolución de problemas sociales los más demandados, por conectar el pasado con el presente.

Palabras clave: contenidos históricos; maestros en formación; Educación Primaria; currículo, temas sociales 


\begin{abstract}
The aim of this work is exposing an analysis of the historical contents that students of the bachelor's degree in Primary Education would like to teach when they become teachers in a school. First, the impact of new historiographical trends in the teaching of History is studied, and if they are of interest to future teachers. Through a sample of 118 participants from the Complutense University of Madrid, with forms that offer quantitative and qualitative data, we obtain significant results on the future of History teaching. These results reveal a profound division between the curricular contents and the motivation of the students who want to teach certain topics, being the resolution of social problems the most demanded, for connecting the past and the present.
\end{abstract}

Key words: historical contents; teachers in training; Primary School; curriculum, social issues. 


\section{Introducción}

Desde que en el siglo XIX en España se configurase la Historia como una materia a impartir en las escuelas públicas, el Estado utilizó su enseñanza para crear un sentimiento de identidad nacional, construido sobre un pasado común (Maestro, 2002; Valls, 2018). Esto continuó a principios del siglo XX y desde luego durante todo el régimen franquista, favorecido por el monopolio de una historiografía positivista a la hora de plantear cómo enseñar Historia. Sujetos históricos, cuyas hazañas heroicas ensalzaban las glorias del Estado, sirvieron de hilo conductor del aprendizaje histórico durante décadas (Gómez Carrasco y Rodríguez Pérez, 2017). Aprendizaje que, por otro lado, carecía de didáctica, obligando al alumnado a un método tradicional como es la memorización de los datos.

En las últimas décadas del siglo XX, a la enseñanza de la Historia se le han reconocido otras funciones, siendo la más notable, la de ayudar a interpretar el presente y a madurar el pensamiento crítico de los estudiantes. Este cambio ha sido posible, entre otras cosas, gracias a la irrupción de nuevas corrientes historiográficas que llegaron para quedarse, y que han permitido la enseñanza más reflexiva acompañada de nuevos contenidos que incluían a más sujetos históricos que el omnipresente "hombre blanco occidental". Además de otros grupos sociales, también se le han sumado otros temas y escenarios (Fontana, 2004). Nuevos temas y también metodologías didácticas más recientes que, en muchos casos, han permitido conectar el presente con el pasado y buscar una base histórica a problemas sociales de nuestra actualidad (Prats y Santacana, 2011; López Facal, 2002; Gómez Carrasco et al., 2015).

Entre las corrientes historiográficas más utilizadas en España a la hora de enseñar Historia, que se siguen considerando recientes, aunque algunas de ellas tienen más de cuarenta años de antigüedad, destaca la historia de la vida cotidiana, que nos acerca a entornos más privados, familiares, domésticos, rurales o urbanos, y a actividades más distendidas en el tiempo (permanencias), incluso, al interés en otras categorías sociales como la niñez, la vejez, la pobreza o la esclavitud (Hernández Sandoica, 2007). Como categoría más general, pero en conexión con las anteriores, encontramos la historia de las mentalidades, muy vinculada también a otras disciplinas como la antropología, que permitió el estudio de cuestiones como el matrimonio, la sexualidad o las emociones (Alcaraz y Pastor, 2012). En esta misma línea, sobresalen los esfuerzos de la historiografía del periodo de la transición española (aproximadamente 1975-1980) por introducir en las aulas el estudio de la microhistoria a través de la historia regional, o más 
aún, de la historia local, como reacción a una historia nacional y patriótica de la época franquista (García Cárcel, 2001). La historia regional y local ha permitido que el alumnado ponga en valor el entorno social, natural y patrimonial de su pueblo, ciudad o región. Sin duda, la que goza de mejor salud y apoyo, debido al empuje del movimiento feminista, es la historia de las mujeres y los estudios de género, que han puesto voz a la historia de la otra mitad de la población (Rausell, 2017; Rugna, 2014; Segura, 2015). Otra corriente a destacar en España, en las últimas décadas, y siguen en auge, son los nuevos planteamientos historiográficos y didácticos que se han puesto al servicio de la educación cívica, fomentando así la mejora de los valores sociales, como la tolerancia, el respeto a la diversidad cultural o la igualdad. Se trata de una historia social y cultural que permite al alumnado asomarse al pasado de minorías sociales, de otros grupos culturales, o que debata en el aula sobre el origen de las migraciones (Alba y Navarro, 2018).

No obstante, es una realidad que la mayoría de los estudiantes que quieren ser maestros y maestras llegan a la Universidad con un recuerdo muy tedioso de los contenidos de Historia aprendidos en la escuela (Montaña, 2014). La bibliografía que se refiere a este tema nos presenta un panorama bastante negativo pues el alumnado que se prepara para ser docente de Primaria en España tiende a reproducir en su puesto de trabajo aquel modelo tradicional que conoció en la escuela, y que como docentes en prácticas siguen observando en otros maestros y maestras que continúan aferrándose a una Historia factual, basada en fechas y nombres de personajes de corte político-institucional. Ese mismo modelo tradicional es el que la estructura curricular, tal y como está organizada en España, poco ayuda a renovar (Pagès, 1999). Si a todo esto, se le suma una escasa innovación metodológica a la hora de enseñar Historia, bien por falta de tiempo, por falta de recursos, etc., el aprendizaje entra en espiral, limitándose a una todavía muy presente memorización de datos en las aulas (Gómez Carrasco et al., 2018; Montaña, 2015). Es difícil hacer entender al alumnado que se está formando en el grado de Educación Primaria que la Historia que se enseña, tiene una utilidad, y que su enseñanza queda lejos de la mera exposición de fechas y nombres, y que las guerras y las conquistas no son las únicas que generaron cambios históricos. Todavía más complicado es hacerles comprender que la Historia no es un bloque de hormigón, sino que es un ente vivo; la Historia puede y debe ser cuestionada, tiene múltiples interpretaciones y debe ser planteada desde distintas perspectivas. Resulta sorprendente que se sigan repitiendo esquemas obsoletos en las aulas de Primaria de los colegios españoles con la abundante 
bibliografía a nuestro alcance sobre nuevas tendencias historiográficas, recursos y metodologías docentes, publicada en las últimas décadas (Maestro, 2002). En este sentido, la educación universitaria tiene la responsabilidad de seguir colaborando para conseguir romper este círculo vicioso, enseñando a los futuros maestros y maestras distintas corrientes historiográficas y nuevos contenidos, además de metodologías y recursos, que amplíen y mejoren los temas a enseñar y aprender.

Hoy en día existe una fuerte contradicción, cuando un maestro o maestra en formación del grado de Educación Primaria aprende a navegar entre distintas corrientes historiográficas, y se enfrenta al ejercer su profesión a un currículo aparentemente rígido, poco flexible, cuyo aprendizaje de contenidos sigue siendo eminentemente factual. De hecho, se dibuja un modelo neopositivista del currículo que busca recuperar valores patrióticos a través de las hazañas gloriosas de sus héroes (López Facal, 2014).

Exponemos a continuación una tabla en la que se muestran de manera sintetizada y resumida los contenidos históricos que aparecen en el Decreto 89/2014, de 24 de julio, por el que se establece el currículo de Educación Primara para la Comunidad de Madrid (CAM), que los toma a su vez de los contenidos curriculares propuestos con anterioridad por el Ministerio de Educación, Cultura y Deporte (RD. 126/2014, de 28 de febrero), y que, como se puede comprobar, no tienen en cuenta en su enunciado curricular al empuje de nuevas corrientes historiográficas (Tabla 1, pág. 78).

Si tomamos, por ejemplo, algunos de los contenidos que se tratan más detalladamente en el currículo de la CAM para $4^{\circ}$ curso de Primaria (D. 89/2014, de 24 de julio), en concreto para el estudio de la Hispania romana, vemos, que se exige identificar la fecha del desembarco romano en la Península, el nombre de Hispania dado a la Península Ibérica por los romanos y conocer algunas hazañas de personajes que se presuponen los más representativos de la época (Escipión, Viriato y Julio César).

Este mismo esquema se repite en el estudio de otras épocas como Al-Ándalus ( $5^{\circ}$ curso), en donde se pide en particular identificar el momento de llegada de los musulmanes, el nombre dado por los musulmanes a la Península Ibérica bajo su dominio, y conocer los nombres de personajes andalusíes como Abd al-Rahman I, Abd al-Rahman III o Averroes. Curiosamente de estos tres personajes no se especifica que se aprendan sus gestas, como sí ocurría con los tres de Hispania, dejando a la vista cierto halo patriótico y un trato desigual dependiendo de la cultura a estudiar; a los romanos se les identifica 
como propios de nuestra cultura pasada, pero no así a los musulmanes que se establecieron en la Península.

Tabla 1. Síntesis de los contenidos históricos propuestos en el currículo de Madrid¹.

\author{
$3^{\circ}$ PRIMARIA \\ Introducción al tiempo histórico: \\ - El siglo y los años como unidades de medida del tiempo histórico. \\ - Números romanos para indicar los siglos. \\ - Datación del tiempo histórico (a.C, d.C, edad y período). \\ - Las fuentes históricas y su clasificación. La Arqueología. El Patrimonio histórico, \\ cultural y artístico.
$4^{\circ}$ PRIMARIA
Prehistoria Península Ibérica:
- La Península Ibérica en la Prehistoria.
- Yacimientos. Atapuerca y Altamira.
- Transformaciones en el Paleolítico, el Neolítico y la Edad de los Metales.
Edad Antigua en la Península:
- Culturas prerromanas autóctonas de la Península Ibérica (celtas e iberos).
- La conquista romana de la Península Ibérica. La Romanización de Hispania.
- La caída del Imperio romano. Invasiones bárbaras. El fin de la Edad Antigua. \\ - Los pobladores colonizadores del Mediterráneo (fenicios, griegos y cartagineses).

\section{$5^{\circ}$ PRIMARIA} \\ Edad Media en la Península Ibérica: \\ - La Hispania visigoda. Los musulmanes en la Península Ibérica. \\ - Los reinos cristianos en la Edad Media. La Reconquista. La sociedad feudal. \\ Camino de Santiago. Reinos cristianos durante la Reconquista en el mapa. Don \\ Pelayo y el Cid Campeador. Principales Reyes castellano-leoneses y aragoneses. \\ Batalla de las Navas de Tolosa (1212). \\ Edad Moderna en España (ss. XVI-XVIII): \\ - Los Reyes Católicos. Origen del Reino de España. Conquistadores, navegantes y \\ exploradores. Conquista de Granada. Colón y América. \\ - Siglos XVI y XVII. El reinado de la Casa de Austria. El Siglo de Oro. \\ - El siglo XVIII. Los Borbones en España. Expediciones científicas de Jorge Juan. \\ Reinado de Carlos III.
}

\title{
$6^{\circ}$ PRIMARIA
}

Edad Contemporánea (del s. XIX al s. XXI):

- Primera mitad del siglo XIX. Guerra de la Independencia (1808-1814) contra la ocupación francesa y el ejército de Napoleón Bonaparte. La primera Constitución liberal de España (Cádiz, 1812). Guerras Carlistas. Francisco de Goya.

- El sistema de la Restauración (1875-1923). Reyes Alfonso XII y Alfonso XIII, Antonio Cánovas del Castillo y escritores Benito Pérez Galdós y Pío Baroja. 1898, pérdida de las colonias.

- Siglos XX y XXI. La República, la Guerra Civil y el franquismo. Actual democracia: la Constitución Española (1978), la incorporación de España a la Comunidad Económica Europea (1986) y la entrada del euro como moneda corriente (2002). 
Es una realidad que las escuelas historiográficas más recientes, como la historia de las mujeres o el análisis de la vida cotidiana, que exponíamos anteriormente, apenas han sido tenidas en cuenta a la hora de estructurar los contenidos históricos del currículo.

Tabla 2. Personajes históricos en el currículo de Educación Primaria de la Comunidad Autónoma de Madrid $^{2}$.

\begin{tabular}{|c|c|c|c|}
\hline $\begin{array}{l}\text { Militares y } \\
\text { gobernadores }\end{array}$ & $\begin{array}{l}\text { Filósofos y } \\
\text { Escritores/as }\end{array}$ & Pintores & $\begin{array}{l}\text { Navegantes, } \\
\text { conquistadores } \\
\text { y exploradores }\end{array}$ \\
\hline Escipión & Séneca & El Greco & Hernán Cortés \\
\hline Viriato & Averroes & Velázquez & Pizarro \\
\hline Julio Cesar & Garcilaso de la Vega & Goya & Magallanes \\
\hline Trajano & Santa Teresa de Jesús & & Elcano \\
\hline Adriano & San Juan de la Cruz & & Cristóbal Colón \\
\hline Rómulo Augusto & Fray Luis de León & & Jorge Juan \\
\hline Adbderramán I & Cervantes & & \\
\hline Abderramán III & Lope de Vega & & \\
\hline Pelayo & Góngora & & \\
\hline Cid Campeador & Tirso de Molina & & \\
\hline Alfonso VI & Calderón de la Barca & & \\
\hline Fernando III & El Greco & & \\
\hline Alfonso X & Velázquez & & \\
\hline Jaime I & Goya & & \\
\hline Isabel de Castilla & Pérez Galdós & & \\
\hline Fernando de Aragón & Pío Baroja & & \\
\hline Carlos I & & & \\
\hline Felipe II & & & \\
\hline Felipe IV & & & \\
\hline Felipe V & & & \\
\hline Carlos III & & & \\
\hline Napoleón Bonaparte & & & \\
\hline Alfonso XII & & & \\
\hline Alfonso XIII & & & \\
\hline Cánovas del Castillo & & & \\
\hline
\end{tabular}

Pongamos un ejemplo muy visual: si hacemos un rápido repaso de los contenidos del currículo de E. Primaria de la Comunidad de Madrid, en el área de Historia que se conoce como la Huella del tiempo, que comienza en $3^{\circ}$ de Primaria ( $8-9$ años) hasta $6^{\circ}$ de Primaria (11-12 años), en la que se trata de aprender la Historia de España, podemos observar la gran importancia que todavía hoy tiene el estudio de los personajes históricos y sus hazañas. Y más aún, la escasa importancia otorgada al conocimiento de personajes femeninos de la Historia de España. De un total de 49 personajes señalados como relevantes para su estudio porque aparecen expresamente citados en el currículo (Tabla 2), sólo 2 figuras son femeninas: Isabel la Católica y Santa Teresa de Jesús (el modelo histórico de una reina y una monja).

2 Fuente: elaboración propia. 
Por tanto, a pesar de la abundante bibliografía (Fernández Valencia y Díez Bedmar, 2019) y de las reivindicaciones y exigencias sociales, se sigue silenciando la historia de otras mujeres que también fueron referentes de la Historia de España; artistas, pintoras, escritoras, humanistas, pensadoras, filósofas, etc. Ante el presente currículo parece difícil plantear el estudio de las actividades de las mujeres a lo largo de la Historia, no sólo en su faceta pública, que la tuvo, sino también como mantenedora de los lazos familiares y grupales (Hernando, 2018).

\section{Método}

El objetivo principal de este estudio es comprobar si el interés de los futuros docentes de Educación Primaria en impartir unos u otros contenidos de Historia se alejaba de los temas que rigen el currículo de Primaria. En cuanto a los objetivos específicos perseguíamos los siguientes:

1. Identificar los contenidos de mayor interés que enseñarían el profesorado en formación en la materia de Historia para Primaria.

2. Detectar los contenidos históricos mejor valorados por los participantes.

3. Caracterizar los contenidos que los participantes propondrían enseñar cuando impartieran Historia en Primaria.

Para ello, se ha utilizado un instrumento de medición de carácter cuantitativo no experimental tipo formulario, que nos ha permitido analizar múltiples factores que inciden en la preferencia por la impartición de unos u otros contenidos históricos (Castro et al., 2015).

\subsection{Muestra}

Para esta investigación se recogió una muestra de 118 estudiantes (16 chicos y 102 chicas) que cursaron en tres grupos distintos la asignatura de Fundamentos y Didáctica de la Historia en la Facultad de Educación de la Universidad Complutense de Madrid en el curso 2019-2020. Es una asignatura obligatoria de $2^{\circ}$ curso del grado de Educación Primaria. Su edad oscila entre los 19 y 21 años (97\%) y los 22-26 años (3\%). Este alumnado veía por primera vez una materia dedicada a la enseñanza de las Ciencias Sociales en su experiencia de formación como futuros maestros. Los 118 participantes eran, naturalmente, una reducida muestra del alumnado de Educación Primaria, no obstante, son también un estudio de caso que puede ofrecer un panorama 
significativamente amplio y diverso del modo de entender la enseñanza de los contenidos históricos.

\subsection{Instrumento}

Elaboramos un instrumento de recogida de datos anónimo que se completaba en dos fases: en la primera se les presentó un formulario con un listado elaborado ad hoc, en el que debían elegir 7 de los 14 ítems que se les ofrecía y, a la vez, debían ordenar de mayor a menor importancia los 7 ítems elegidos. Este instrumento nos permitió identificar las contradicciones entre el currículo y lo que los docentes querían enseñar teniendo en cuenta distintas variables: sus inquietudes, el valor que le dan a unos u otros contenidos históricos a la hora de ser enseñados en Primaria, y la propia experiencia formativa de los participantes en sus etapas escolares. El listado ofrecía un total de 14 ítems con contenidos de Historia de distintas escuelas historiográficas (antiguas y más recientes) y les propusimos el siguiente formulario (A):

A) Ya eres maestro/a de E. Primaria y para la parte de Historia de la asignatura de Ciencias Sociales, debes decidir qué contenidos históricos quieres enseñar. Elige 7 ítems entre los 14 que se te proponen, y ordena los 7 según el nivel de importancia para ti; pensando en tu motivación, en tu propia experiencia escolar y en el interés del alumnado al que impartirás docencia.

- $\square$ Los hechos más relevantes de la $H^{a}$ de España

- $\square$ La diversidad cultural existente en nuestra sociedad

- $\square$ Las etapas de la $H^{a}$ de España y sus características fundamentales

- $\square$ Los derechos y responsabilidades de los/as españoles/as en una sociedad plural

- $\square$ Los descubrimientos e inventos españoles que han contribuido al progreso humano

- $\square$ La historia de las mujeres en España

- $\square$ Las batallas y guerras que cambiaron la $H^{a}$ de España y sus consecuencias

- $\square$ Los personajes más significativos de la $H^{a}$ de España y su contribución

- $\square$ Las características propias de nuestra identidad

- $\square$ La organización política de España respecto al mundo

- $\square$ La mezcla cultural en los encuentros entre sociedades (Ej. Los españoles y América)

- $\square$ La historia de otras categorías sociales en España (la infancia, la vejez, la esclavitud, la élite)

- $\square$ Los cambios y permanencias en la vida cotidiana de la $H^{a}$ de España

- $\square$ Los problemas sociales del país en el que vivimos acudiendo a su origen en el pasado 
En una segunda fase, terminado el formulario con el listado anterior, se les propuso a los participantes que elaboraran una propuesta (B):

B) Elabora un ítem nuevo si piensas que falta alguna temática en esta lista porque consideras que es relevante su aprendizaje.

\subsection{Procedimiento de recogida y análisis de datos}

Tanto el formulario-listado A como la propuesta B se realizaron el primer día de clase en el mes de septiembre, antes incluso de presentar la asignatura en los tres cursos, para que no se sintieran cohibidos por el temario de las guías docentes, y se pudieran expresar con mayor libertad. Por otra parte, se trataba también de contar con su experiencia cuando estudiaban la asignatura de Historia en el colegio e instituto. Si este estudio lo hubiéramos realizado al término de la asignatura, probablemente los resultados habrían sido distintos, y simplemente estaríamos valorando nuestro método docente, que no es el interés de este artículo en el que tratamos de conocer el interés de los estudiantes de Educación Primaria en unas u otras corrientes historiográficas, lo que se traduce en la elección de temas que les gustaría tratar cuando sean docentes.

El instrumento fue aplicado por tres profesores del área de didáctica de las Ciencias Sociales, informando previamente a los participantes de la finalidad del estudio. El procedimiento presentaba una metodología activa, que generó gran motivación, y que derivó en un debate al término del formulario, con lo que se consiguió sensibilizar a los estudiantes y poner en cuestionamiento las bases historiográficas que hoy en día sustentan el currículo en el área de Historia.

La dificultad para analizar los datos radicaba en la interpretación del orden de los ítems elegidos por los participantes (del $1^{\circ}$ al $7^{\circ}$ lugar) en relación con su importancia. No obstante, nos ha permitido analizar mejor la complejidad del problema, porque ordenaba sus prioridades y nos proporcionaba resultados sugerentes, como se comprobará en el análisis de los resultados. Los datos obtenidos se interpretan a través de dos figurasgráficas; por un lado, en la primera se trabaja el orden de elección de los ítems elegidos, por el otro, en la segunda se presentan los temas mejor y peor valorados por los participantes. Se añade una tabla con nuevos contenidos propuestos por los participantes que consideran relevantes para ser impartidos en Primaria, y que no aparecían en el listado que les ofrecíamos de partida en el formulario. Las respuestas de esta propuesta que 
dieron los participantes, en algunos casos, fueron clasificadas en categorías, dado que hacían referencia a temas similares, pero usando un vocabulario distinto, de manera que la interpretación queda así más clara.

Por último, tras cumplimentar los cuestionarios realizamos un debate con el alumnado para aclarar algunas dudas y cuestiones o ampliar el significado de algunas de sus respuestas. Apuntes, que se incluye en el análisis de datos para dar explicación a algunos de los resultados.

\section{Resultados y Análisis de datos}

\subsection{Contenidos de mayor interés que enseñarían los futuros docentes en Historia}

A continuación, mostramos los resultados (Figura 1) obtenidos del primer formulariolistado.

Figura 1. Contenidos elegidos por maestros/as de Educación Primaria en formación.

Los cambios y permanencias en la vida cotidiana de la $\mathrm{H}^{\mathrm{a}}$ de España

La historia de otras categorías sociales en

España (la infancia, la vejez, la...

La mezcla cultural en los encuentros entre sociedades (Ej. Los españoles y América)

La organización política de España respecto

$$
\text { al mundo }
$$

Las características propias de nuestra

$$
\text { identidad }
$$

Los personajes más significativos de la $\mathrm{H}^{\mathrm{a}}$ de España y su contribución

Las batallas y guerras que cambiaron la $\mathrm{H}^{\mathrm{a}}$ de España y sus consecuencias

La historia de las mujeres en España

Los descubrimientos e inventos españoles que han contribuido al progreso humano

Los derechos y responsabilidades de los/as españoles/as en una sociedad plural

Las etapas de la $\mathrm{H}^{\mathrm{a}}$ de España y sus características fundamentales

La diversidad cultural existente en nuestra sociedad

Los hechos más relevantes de la $\mathrm{H}^{\mathrm{a}}$ de España

Los problemas sociales del país en el que vivimos acudiendo al origen en el pasado

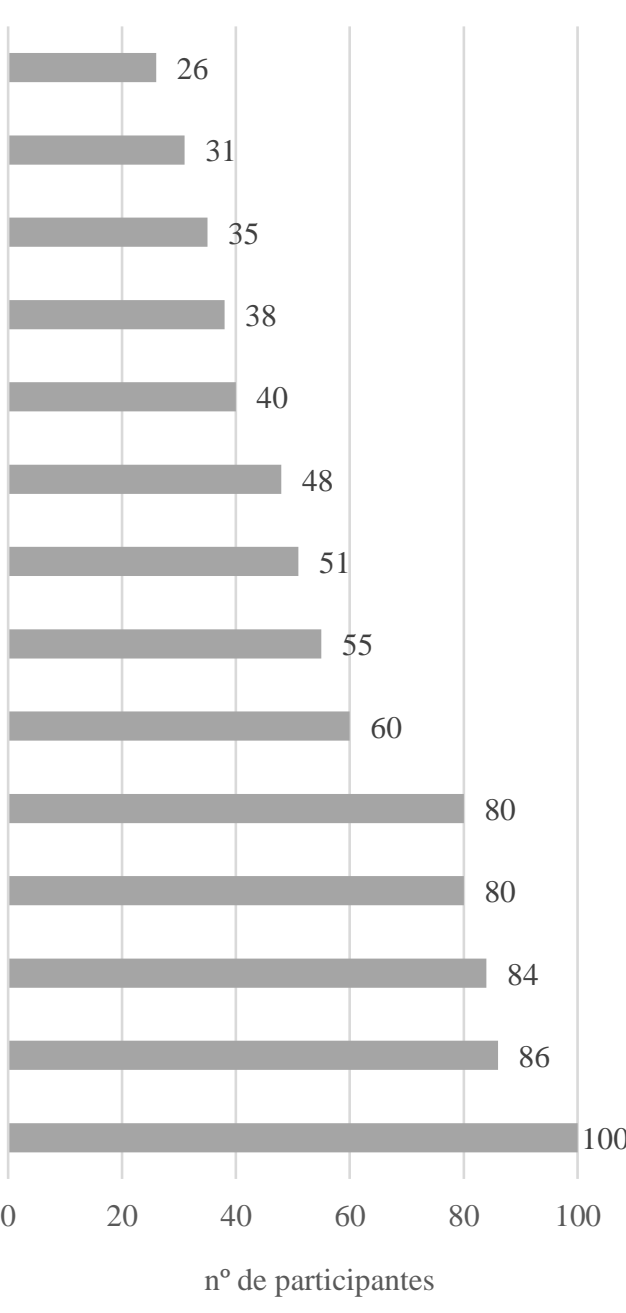


En esta gráfica, aparecen a la izquierda los 14 ítems que presentamos a los participantes con los contenidos históricos de las distintas corrientes historiográficas (antiguas y más recientes). A la derecha, en las barras de color gris, asociado a cada ítem aparece el número total de participantes que lo ha elegido, del total de la muestra que era 118 , independientemente del orden del 1 al 7 que le hayan otorgado a dicho ítem. Se refleja, por tanto, el número de participantes que impartirían esos contenidos en el aula de Educación Primaria. Lógicamente, ningún ítem ha sido seleccionado por el total de la muestra con 118 participantes, porque recordemos que les pedíamos escoger 7 ítems (contenidos históricos) de los 14 que les ofrecíamos, y esto, ha provocado el descarte de la mitad de los ítems por parte de los estudiantes.

Tal y como se puede observar, de los 118 participantes, un total de 100 considera que el estudio de "Los problemas sociales del país en el que vivimos acudiendo al origen en el pasado" no debería faltar en el temario de Historia de España de Primaria. Es decir, un $84.7 \%$ le otorga importancia a este tema, y se plantearía trabajarlo en el aula. Son también considerados necesarios, por más de 80 alumnos/as, cuatro ítems cuyo aprendizaje resulta relevante para la educación escolar, que son: el estudio de "Los hechos más relevantes de la Historia de España" elegido por un total de 86 estudiantes (el 72.8\%); "La diversidad cultural existente en nuestra sociedad" en total por 84 (el 71.1\%); "Los derechos y responsabilidades de los/as españoles/as en una sociedad plural" y "Las etapas de la $\mathrm{H}^{\mathrm{a}}$ de España y sus características fundamentales", en el que cada uno ha sido considerado por 80 estudiantes (67.7\%). Si recapitulamos la información, en orden de interés, primero aparecen los problemas sociales, después los hechos más relevantes, le siguen la diversidad cultural y las etapas de la Historia de España. Este resultado permite valorar la importancia que nuestros estudiantes otorgan a la cronología en la enseñanza de la Historia, así como la necesidad de tratar temas que permitan entender mejor a la sociedad actual, con temas cívicos y éticos como la búsqueda del origen a los problemas sociales, y reconocer la pluralidad cultural que existe en España (Cernadas et al., 2019). Esto último puede estar motivado por la presencia cada vez más importante de la competencia social y cívica en el currículum y en los libros de texto, que refuerza todos los aspectos relativos a los derechos y deberes de los ciudadanos, el respeto a la Constitución, la tolerancia, etc., a lo largo de toda la enseñanza obligatoria.

En cuanto a los ítems peor valorados, con menos de cuarenta participantes interesados en estos contenidos, aparece "Los cambios y permanencias en la vida cotidiana de la $\mathrm{H}^{\mathrm{a}}$ de 
España", con un total de 26 estudiantes que lo han valorado con algún punto (22\%), "La historia de otras categorías sociales en España (la infancia, la vejez, la esclavitud)" votada por 31 participantes (26.2\%), o "La mezcla cultural en los encuentros entre sociedades (Ej. Los españoles y América)" puntuada por 35 estudiantes (29.6\%). Por último, además de estos tres ítems de corte social y cultural, aparece poco considerado el enseñar "La organización política de España en relación con el mundo" (38 participantes que suponen un $32.2 \%$ ). El escaso interés que muestran los participantes en la vida cotidiana y en el estudio de nuevas categorías sociales como la infancia o la vejez deriva de su desconocimiento y de la poca atención que le han dedicado en su etapa escolar tanto en Primaria como en Secundaria, tal y como algunos estudiantes nos expresaron al evaluar los resultados entre todos. También nos aclararon que, el escaso valor dado a la enseñanza de la organización política de España en relación con el mundo deriva de que la materia a impartir es a nivel nacional en Primaria y les parecía fuera de los límites del temario una comparativa política a nivel mundial. Además, nos indicaron que la historia política estaba incluida en los hechos más relevantes y en las etapas de la Historia de España, siendo la política y diplomacia el único hilo conductor de estos temas.

Por último, entre 40 y 60 participantes han considerado, como importantes, temas como la historia de las mujeres (46.6\%), los personajes históricos (40.6\%), las batallas y guerras (43.2\%), y los descubrimientos $(50.8 \%)$.

\subsection{Contenidos históricos mejor valorados por los participantes}

Los resultados de la Figura 2 nos permiten valorar los ítems mejor calificados de la lista; que han sido elegidos por los participantes en $1^{\circ}$ lugar. A la izquierda, los 14 ítems, de los que recordamos que cada participante escogió sólo 7, y a la derecha contrastamos dos datos diferenciados por colores: en gris claro, los datos de la gráfica anterior, con el total de participantes que eligieron cada ítem, sin tener en cuenta el orden de predilección, y en gris oscuro, el número de participantes que eligieron ese mismo ítem en $1^{\circ}$ lugar. El hecho de contrastar las dos informaciones nos permite valorar si los ítems elegidos por el mayor número de participantes (gris claro) se corresponden con los ítems mejor valorados (gris oscuro), considerados como materia imprescindible a impartir por nuestros estudiantes cuando ejerzan de maestros. Así se contrastan mejor los resultados de la gráfica anterior con los de la nueva. 
Figura 2. Contenidos históricos mejor valorados, colocados en $1^{\circ}$ lugar por los participantes.

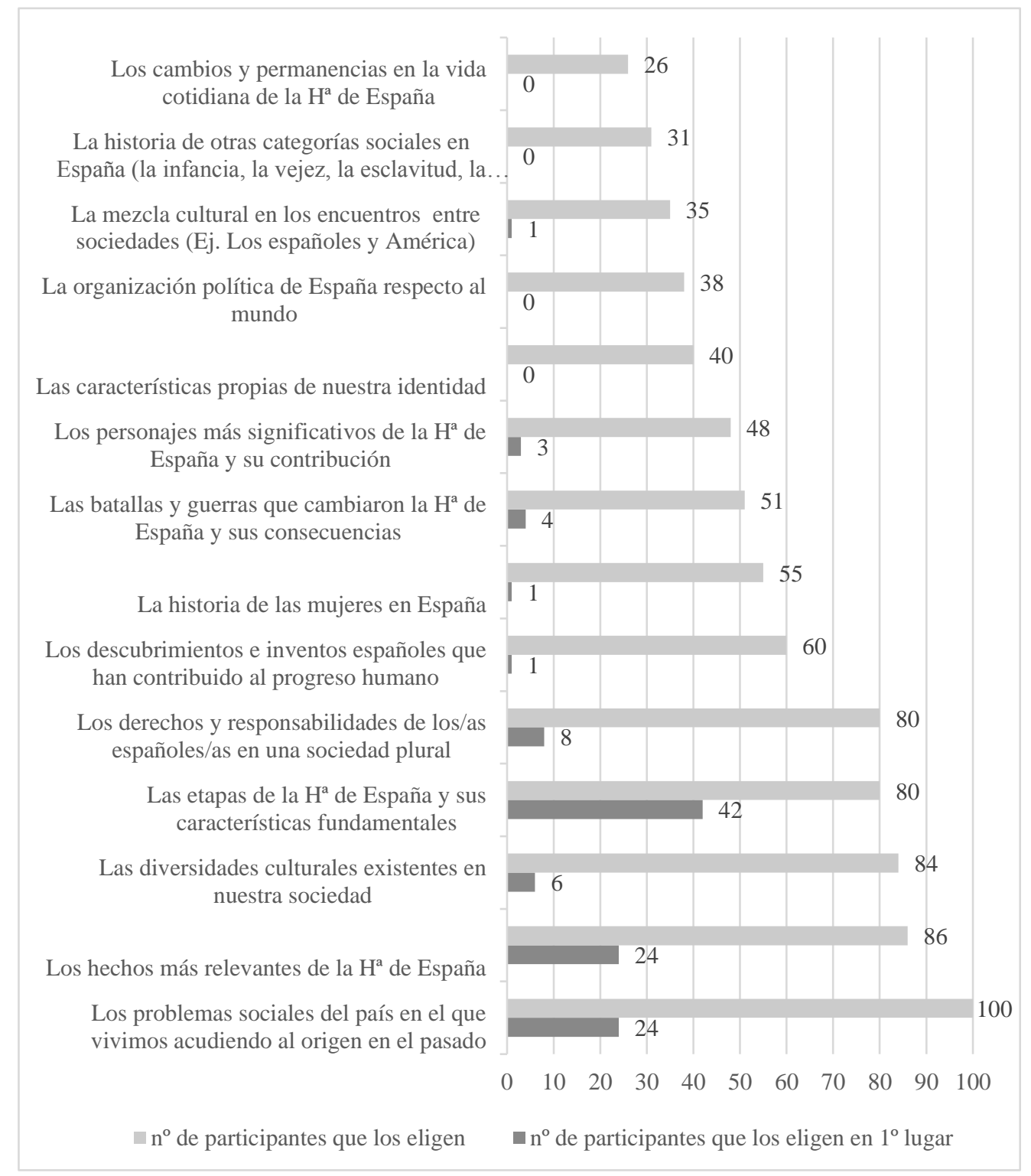

De los datos podemos extraer que el ítem mejor valorado ha sido el aprendizaje de "Las etapas de la Historia de España y sus características fundamentales", considerado por 42 participantes de los 118 (35.5\%) como la cuestión más importante a enseñar en Primaria. Ahora bien, el ítem que anteriormente sobresalía por ser el elegido por más estudiantes (100 de 118) era "Los problemas sociales del país en el que vivimos acudiendo al origen en el pasado"; sin embargo, destaca que sólo 24 estudiantes lo hayan colocado en primer lugar (un 20.3\%). Esto se traduce en que la mayoría de los participantes pondrían atención en trabajar los problemas sociales desde una perspectiva histórica, pero que sólo unos pocos lo considerarían primordial, a diferencia de "Las etapas de la Historia de España y sus características fundamentales" que se sitúa en la cúspide de las cuestiones a aprender 
en Ciencias Sociales de Primaria. Ocurre algo parecido a la hora de elegir trabajar el tema de "La diversidad cultural existente en nuestra sociedad", que muchos participantes la escogieron, 84 de 118 (71.1\%), pero apenas 6 estudiantes (5\%) consideran estos contenidos como los más importante a desarrollar en las clases de Historia de Primaria.

Ningún participante ha colocado en primer lugar ni "Los cambios y permanencias en la vida cotidiana de la $\mathrm{H}^{\mathrm{a}}$ de España", ni "La historia de otras categorías sociales en España (la infancia, la vejez, la esclavitud)", ni "La organización política de España en relación con el mundo", ni "Las características propias de nuestra identidad". Consideran que son temas secundarios en las clases de Historia, incluso innecesarios de aprender, en consonancia con los datos que aportaba la anterior gráfica. De nuevo, nos relataban el desconocimiento que presentan hacia historiografías más recientes, sin entender para qué sirve saber, por ejemplo, qué si un niño en la época medieval iba o no a la escuela, tal y como nos justificó un estudiante durante el debate.

\subsection{Contenidos de Historia que los participantes propondrían enseñar}

Aquí se presenta el análisis de los resultados en relación con la segunda fase del instrumento, que proponía lo siguiente:

B) Elabora un ítem nuevo si piensas que falta alguna temática en esta lista porque consideras que es relevante su aprendizaje.

Hemos unificado respuestas similares, dando como resultado la Tabla 1 (pág. 78). Estos datos son interesantes porque ya no les ofrecíamos las opciones temáticas, sino que ellos proponían unos contenidos que consideraban motivadores y útiles para la enseñanza de la Historia.

Hay que tener en cuenta que los resultados en número y porcentaje parecen bajos, pero es debido a una atomización de los temas, siendo muy variados los ítems propuestos por los participantes, y de éstos, algunos sugeridos tan sólo por uno o dos estudiantes. Es importante tener en cuenta que les pedíamos que pensaran en su motivación y en la de su futuro alumnado de Educación Primaria; en los temas que ellos consideraban que habían sido olvidados, casi nada tratados o invisibilizados en educación y que les gustaría enseñar en Historia de España (de $3^{\circ}$ a $6^{\circ}$ de Primaria). 
Tabla 1. Contenidos históricos que les gustaría explicar en Educación Primaria.

\begin{tabular}{|c|c|c|}
\hline Propuestas del alumnado & $\mathbf{N}^{\mathbf{o}}$ & $\%$ \\
\hline Historia de la Comunidad o Provincia en la que vives & 21 & 17.8 \\
\hline $\begin{array}{l}\text { Consecuencias del pasado que se evidencian en la actualidad de } \\
\text { España }\end{array}$ & 18 & 15.2 \\
\hline $\begin{array}{l}\text { Manifestaciones artísticas, artistas y su influencia en la sociedad } \\
\text { española }\end{array}$ & 13 & 11 \\
\hline La capacidad de progreso de los españoles en el tiempo & 8 & 6.7 \\
\hline Historia del género: comparativa hombres-mujeres en España & 8 & 6.7 \\
\hline Educación en valores a través de la $\mathrm{H}^{\mathrm{a}}$ de España & 6 & 5 \\
\hline Migraciones en el pasado de España & 6 & 5 \\
\hline Desigualdad social en España y acciones solidarias & 5 & 4.2 \\
\hline Tradiciones, leyendas y misterios en España & 5 & 4.2 \\
\hline Reflexión crítica de los principales hechos históricos & 4 & 3.4 \\
\hline Historia de la Educación española & 4 & 3.4 \\
\hline $\begin{array}{l}\text { El uso de las fuentes y búsqueda de información en archivos y } \\
\text { bibliotecas }\end{array}$ & 3 & 2.5 \\
\hline Evolución del trabajo y su organización social en España & 3 & 2.5 \\
\hline $\begin{array}{l}\text { Interdisciplinariedad entre Historia, Literatura, Arte y } \\
\text { Antropología }\end{array}$ & 3 & 2.5 \\
\hline Evolución de la globalización & 3 & 2.5 \\
\hline Evolución de la tecnología en España y en el mundo & 2 & 1.7 \\
\hline $\begin{array}{l}\text { Historia del cine, televisión y música en el mundo y su } \\
\text { repercusión en España }\end{array}$ & 2 & 1.7 \\
\hline $\begin{array}{l}\text { Historia del origen de las religiones: cristianismo, judaísmo e } \\
\text { islam }\end{array}$ & 2 & 1.7 \\
\hline $\begin{array}{l}\text { Historia de personajes menos conocidos que generaron cambios } \\
\text { sociales }\end{array}$ & 1 & 0.8 \\
\hline Historia de la familia & 1 & 0.8 \\
\hline
\end{tabular}

Resulta interesante que un total de 21 participantes (17.8\%) den importancia a una "Historia de la Comunidad o Provincia en la que se vive" porque, como nos señalaban, no están acostumbrados en el currículo de la Comunidad Autónoma de Madrid (D. 89/2014, de 24 de julio) a dar importancia a este tema. Algún apartado específico de la Historia de Madrid con los Austrias y Borbones aparecía en sus manuales de Historia cuando estudiaban, pero de manera anecdótica, o a modo de curiosidad, al final del tema de Edad Moderna. Y esto continúa hoy en día en las editoriales de Ciencias Sociales para Primaria. Madrid es de las provincias de España que menos interés pone en enseñar la historia de la ciudad porque algunos estudiantes de otras comunidades (a través del programa de movilidad del Sistema de Intercambio entre Centros Universitarios Españoles, SICUE) expresaban que en Primaria en sus regiones se incide mucho en la historia local. Y así lo corroboran otros currículos como el de la región de Murcia (D. 198/2014, de 5 de septiembre) o el de Andalucía (D. 97/2015, de 3 de marzo). Los 
participantes de este estudio consideran importante conocer la historia de Madrid porque el aprendizaje en didáctica se entiende de lo cercano a lo lejano. Por otra parte, una mayor presencia curricular de la historia de su ciudad les permitiría realizar excursiones, así como poner en marcha recursos didácticos de revalorización del patrimonio local. Esta falta de sensibilidad hacia la historia de Madrid encuentra su explicación en que dicha ciudad ha sido un espacio que hasta época moderna no fue relevante en la Historia de España. Hasta que Felipe II no situó la corte en ella, en 1561, Madrid no ha destacado, y desde entonces, siempre ha alojado (excepto en momentos muy puntuales que se trasladó la corte a otros lugares) el gobierno central, reduciendo el interés de la perspectiva regional en favor del interés nacional.

Una mirada crítica al pasado que han denominado los estudiantes como "Consecuencias del pasado que se evidencian en la actualidad de España" ha sido elegido como tema interesante que debería ser tratado en la parte de Historia de la asignatura de Ciencias Sociales en Primaria. Lo han propuesto un total de 18 participantes (15.2\%), demandando, por tanto, una mayor reflexión y capacidad crítica a la hora de impartir Historia. También hay que llamar la atención sobre la propuesta de una mayor implicación en temas artísticos y patrimoniales pues 13 participantes (11\%) han planteado que se debería incluir el aprendizaje de "Manifestaciones artísticas, artistas y su influencia en la sociedad española”. En este sentido, señalaban los estudiantes que en Primaria se debería poner más atención a la Historia del Arte, y que no fuera algo residual, porque además de trabajar la educación estética, esta disciplina estimula la creatividad y fomenta valores como el respeto al patrimonio histórico-artístico, a la expresión de las emociones, la empatía, al sentido de la belleza, etc.

Estos tres temas: "Historia de la Comunidad o Provincia", "Consecuencias del pasado en el presente" y "Manifestaciones artísticas..." suman un total de 52 participantes (44\%).

Cabe señalar que los estudiantes apuestan por una "Historia del género", en vez de una Historia de las mujeres, y esto es muy interesante, porque sin la comparativa mujerhombre no se ponen de manifiesto las desigualdades (García Pérez et al., 2010). También es preciso destacar que conceptos como "progreso", "educación en valores", "desigualdad", o "migraciones" son propuestos por entre 5 y 8 participantes (entre $4 \%$ y $6 \%$ ), como un reclamo para que la enseñanza de la Historia sirva para lograr una mejora de la convivencia social. 
Por último, llama la atención en el análisis de los datos, la confusión entre el aprendizaje de contenidos históricos y la adquisición de competencias procedimentales. Esto lo constatamos cuando algunos participantes proponen "el uso de fuentes y búsqueda de información en archivos y bibliotecas", no diferenciando entre metodología y lo que son los contenidos de una asignatura, que es lo que realmente les pedíamos. Esto es algo en lo que se incide en la formación universitaria de nuestro alumnado para que no lleguen a la práctica docente con estas lagunas que comprometen el aprendizaje escolar.

\section{Conclusiones}

Los resultados del análisis de los datos han permitido demostrar la desconexión que existe entre los temas de Historia de España que al alumnado le gustaría enseñar cuando ejerza como maestro/a de Primaria y los que presenta un currículo anclado aún en los temas de historia diacrónica y factual. Esta grieta viene dada por el argumento positivista que continúa ofreciendo el currículo en España para la enseñanza de la Historia, y que los manuales de texto tratan de hacer más asimilable con nuevos recursos didácticos. No obstante, los temas no cambian, y cuesta que nuevas corrientes historiográficas irrumpan en el modelo tradicional, por eso, está en manos de los estudiantes introducir nuevos contenidos que conecten más con la sociedad en la que vivimos, y para ello, hay que darles a conocer nuevas corrientes historiografías para que tengan una mayor variedad de contenidos a tratar.

De los temas que los participantes eligen como más interesantes destacamos los temas que vinculan las problemáticas sociales actuales a procesos del pasado, en definitiva, un uso de la Historia para ampliar y mejorar la comprensión social del presente, tal y como plantea en sus estudios Santiesteban (2019). En este sentido la bibliografía especializada en didáctica de la Historia escucha los intereses del alumnado y les ofrece nuevos temas más atractivos, e incluso más útiles para la convivencia. Sorprende, y es preciso destacarlo en las conclusiones, el poco interés de los estudiantes en enseñar la historia de la vida cotidiana, que refleja la escasa o nula atención que recibe esta corriente historiográfica en los programas de Historia de los colegios. Y esto es una realidad, pues nuestros universitarios no entendían bien qué se puede aprender sobre vida cotidiana y para qué sirve, por eso es necesario enseñar, desde la didáctica de la Historia, aquellas escuelas historiográficas que el currículo y los manuales no incorporan, pero de las que 
los futuros docentes podrán elegir enseñar contenidos históricos, porque dota de relevancia las pequeñas historias, las que conforma la vida de la mayor parte de la población, y desde las que la microhistoria puede aportar numerosos enfoques.

También hay que resaltar que los estudiantes son conscientes de que no se puede descuidar el conocimiento de las etapas que contextualizan nuestra Historia, porque son contenidos determinantes para que los niños y niñas avancen madurativamente en la adquisición de las nociones de temporalidad, vinculadas al tiempo histórico. Tampoco estos maestros y maestras en formación olvidan la importancia del conocimiento de los hechos más relevantes. Sin embargo, demandan más la inclusión de contenidos derivados de corrientes historiográficas más recientes. Por otra parte, cuando les preguntamos qué temas echaban en falta en cuanto a conocimientos históricos, la historia regional o local despunta, siendo un reclamo que el currículo de la Comunidad Autónoma de Madrid debería poner más atención en la historia y patrimonio de la ciudad, como hacen el resto de las comunidades.

Cuando comparamos con nuestros estudiantes los contenidos históricos del currículo de Primaria con los elegidos y propuestos por ellos, se llevaron una gran desilusión al identificar la poca o nada correspondencia entre su motivación o interés y el currículo. Si analizamos los contenidos curriculares de la CAM (D. 89/2014, de 24 de julio) destaca el conocimiento de las Etapas Históricas, y después, saber los hechos más relevantes de nuestra Historia. Asimismo, es importante el estudio de los personajes más significativos de la Historia de España (aunque estos son mayoritariamente masculinos, como se ha señalado).

Por último, el currículo no pone atención a la comprensión de los problemas sociales actuales analizando el pasado; tampoco al conocimiento histórico de las minorías o a la historia de las mujeres. Por lo tanto, no conecta con los intereses de los futuros docentes, y la utilidad del aprendizaje de la disciplina se queda en un mero conocimiento del pasado, sin valorarla adecuadamente como una buena herramienta de progreso social.

No se trata aquí de hacer una crítica a la falta de sensibilidad social de la que adolece el currículo de Primaria en España, pero sí señalar que, la escasa motivación de los futuros docentes en impartir unos contenidos curriculares tradicionales repercute negativamente en la enseñanza de la Historia, así como en el concepto obsoleto que se tiene de la materia. Afortunadamente, más que los manuales de texto, las innovaciones metodológicas en didáctica de la Historia y las nuevas corrientes historiográficas van forzando al currículo 
hacia una mayor inclusión de nuevos contenidos que conecten el pasado con el presente que vivimos.

\section{Financiación}

Este artículo se enmarca en la investigación de los proyectos "Conceptos metodológicos y métodos activos de aprendizaje para la mejora de las competencias docentes del profesorado" (PGC2018-094491-B-C33), financiado por el Ministerio de Ciencia, Innovación y Universidades, y "Madrid, sociedad y patrimonio: pasado y turismo cultural: la vida cotidiana" (MASOPA-CM, Ref. H2019/HUM-5898). Asimismo, forma parte del Proyecto de Innovación Docente "Observatorio de Historia: recursos didácticos para recuperar la memoria de individuos y sociedades invisibilizadas”, (Ref. 103/2021) de la Universidad Complutense de Madrid. 


\section{Referencias}

Alba, N. y Navarro, E. (2018). Concepciones de ciudadanía y educación para la ciudadanía en el profesorado de Educación Infantil y Primaria. En E. López Torres, C.R. García Ruiz y M. Sánchez Agustí (coords.), Buscando formas de enseñar: investigar para innovar en didáctica de las Ciencias Sociales (pp. 491502). Universidad de Valladolid-AUPDCS.

Alcaraz, A. y Pastor, M.M. (2012). Tendencias de la Historia como objeto de enseñanza a través de la Historiografía. Revista de Didácticas Específicas, 6, 114-139.

Castro, L., Castro, M. A. y Morales, J. (2015). Metodología de las Ciencias Sociales. Una introducción crítica. Madrid: Tecnos.

Cernadas, F.X., Lorenzo, M.M. y Santos, M.A. (2019). Diversidad cultural y escenarios migratorios: Un estudio sobre formación de profesores. Educar, 55(1), 19-37.

Decreto 89/2014, de 24 de julio, del Consejo de Gobierno, por el que se establece para la Comunidad de Madrid el Currículo de la Educación Primaria. Recuperado de http://www.madrid.org/wleg_pub/secure/normativas/contenidoNormativa.jsf?o pcion $=$ VerHtml\&nmnorma=8620\&cdestado=P\#no-back-button

García Cárcel, R. (2001). La reciente historiografía modernista española. Chronica nova: Revista de historia moderna de la Universidad de Granada, 28, 185-219.

García Pérez, R., Rebollo Catalán, M.A., Buzón García, O., González-Piñal, R., Barragán Sánchez, R. y Ruíz Pinto, E. (2010). Actitudes del alumnado hacia la igualdad de género. Revista De Investigación Educativa, 28(1), 217-232. Recuperado de https://revistas.um.es/rie/article/view/98951

Gómez Carrasco, C.J., Rodríguez Pérez, R.A. y Miralles Martínez, P. (2015). La enseñanza de la Historia en educación primaria y la construcción de una narrativa nacional. Un estudio sobre exámenes y libros de texto en España. Perfiles $\quad$ Educativos, $\quad 37(150), \quad 20-38$. https://doi.org/10.22201/iisue.24486167e.2015.150.53160

Gómez Carrasco, C.J. y Rodríguez Pérez, R.A. (2017). La historia como materia formativa. Reflexiones epistemológicas e historiográficas. Revista de historiografía (RevHisto), 27, 265-286. 
Gómez Carrasco, C.J., Rodríguez-Pérez, R.A. y Mirete, A.B. (2018). Metodología didáctica y concepciones epistemológicas sobre la enseñanza de la historia. Una investigación con futuros maestros. Revista Complutense de Educación, 24(1), 237-250.

Fernández Valencia, A. y Díez Bedmar, M.C. (2019). Enseñanza de las Ciencias Sociales con perspectiva de género. Clío: History and History Teaching, 45, 1-10.

Fontana, J. (2004). ¿Qué Historia enseñar? Clío\&Asociados, 7, 15-26.

Hernández Sandoica, E. (2007). Nuevas tendencias historiográficas actuales. Madrid: Akal.

Hernando, A. (2018). La fantasía de la individualidad. Dossieres EsF, 30, 6-11.

López Facal, R. (2002). La enseñanza de la historia más allá del nacionalismo. En C. Forcadell Álvarez y J.J. Carreras Ares (coords.), Usos públicos de la historia: ponencias del VI Congreso de la Asociación de Historia Contemporánea (pp. 223-225). Zaragoza: Prensas Universitarias de Zaragoza-Marcial Pons.

López Facal, R. (2014). La LOMCE y la competencia histórica. Ayer, 94(2), 273-285.

Maestro, P. (2002). El modelo de las historias generales y la enseñanza de la Historia. Didáctica de las ciencias experimentales y sociales, 16, 3-33.

Montaña, J.L. (2014). Didáctica de la Historia y profesorado en formación: análisis de concepciones y nuevos paradigmas para una enseñanza-aprendizaje de la Historia. En J. Pagès i Blanch y A. Santisteban (coords.), Una mirada al pasado y un proyecto de futuro: investigación e innovación en didáctica de las Ciencias Sociales (pp. 551-558). Barcelona: Universitat Autònoma de BarcelonaAUPDCS, vol. 2.

Montaña, J.L. (2015). Didáctica de la Historia, Historiografía y la visibilización de temas, grupos sociales y personas en la enseñanza-aprendizaje de la Historia. En A. M. Hernández Carretero, C.R. García Ruiz y J.L. Montaña Conchiña (coords.), Una enseñanza de las ciencias sociales para el futuro: Recursos para trabajar la invisibilidad de personas, lugares y temáticas (pp. 907-915). Universidad de Extremadura-AUPDCS. 
Pagés i Blanch, J. (1999). Las representaciones previas de los estudiantes de los estudiantes de maestro, de Ciencias Sociales, Geografía e Historia. Teoría y Didáctica de las Ciencias Sociales, 4, 161-178.

Prats, J. y Santacana, J. (2011). Didáctica de la Geografía y la Historia. Barcelona: Graó, 2 vols.

Rausell, H. (2017). Ilustración y género. Los modelos de masculinidad presentes en la asignatura de $\mathrm{H}^{\mathrm{a}}$ de España. Didáctica de las ciencias experimentales y sociales, $32,93-108$.

Real Decreto 126/2014, de 28 de febrero, por el que se establece el currículo básico de la Educación Primaria. Ministerio de Educación, Cultura y Deporte. Boletín Oficial del Estado $\mathrm{n}^{\mathrm{o}}$ 52, de 1 de marzo de 2014. Recuperado de https://www.boe.es/buscar/pdf/2014/BOE-A-2014-2222-consolidado.pdf

Rugna, C.M. (2014). Feministas en sus zapatos: historiografía y enseñanza de la historia. Clio \& Asociados. La enseñanza de la Historia, 18, 216-231.

Santiesteban, A. (2019). La enseñanza de las Ciencias Sociales a partir de problemas sociales o temas controvertidos: estado de la cuestión y resultados de una investigación. El futuro del pasado, 10, 57-79.

Segura, C. (2015), “Cómo construimos la Historia de las Mujeres desde las Universidades españolas”. Revista de Historiografía, 22, 255-271.

Valls, R. (2018). Manuales de Historia, entre nacionalismo y crítica: Un estado europeo de la cuestión. En A. Delgado Cendagortagalarza y A. Rivera (eds.), ¿Qué saben de su historia nuestros jóvenes?: Enseñanza de la historia e identidad nacional (pp. 1-6). Granada: Comares. 